\title{
Management of Sigmoid Volvulus Avoiding Sigmoid Resection
}

\author{
Nikolaos Katsikogiannis $^{\text {a }}$ Nikolaos Machairiotis $^{\text {a }}$ \\ Paul Zarogoulidis ${ }^{a, e}$ Eirini Sarika $^{b}$ Aikaterini Stylianaki $^{a}$ \\ Maria Zisoglou ${ }^{a}$ Vasilis Zervas $^{a}$ e Metaxia Bareka $^{f}$ \\ Christos Christofis $^{c} \quad$ Alkis lordanidis $^{d}$ \\ ${ }^{a}$ Surgery Department (NHS), ${ }^{b}$ Biopathology Department, ${ }^{c}$ Anesthesiology \\ Department and ${ }^{\mathrm{d}}$ Radiology Department, University General Hospital of \\ Alexandroupolis, Democritus University of Thrace, Alexandroupolis; \\ ePulmonary Department and ${ }^{\mathrm{f}}$ Anesthesiology Department, 'G. Papanikolaou' \\ General Hospital, Aristotle University of Thessaloniki, Thessaloniki, Greece
}

\section{Key Words}

Acute sigmoid volvulus · Conservative treatment

\begin{abstract}
Acute sigmoid volvulus is typically caused by an excessively mobile and redundant segment of colon with a stretched mesenteric pedicle. When this segment twists on its pedicle, the result can be obstruction, ischemia and perforation. A healthy, 18-year-old Caucasian woman presented to the emergency department complaining of cramping abdominal pain, distention, constipation and obstipation for the last $72 \mathrm{~h}$, accompanied by nausea, vomiting and abdominal tenderness. The patient had tympanitic percussion tones and no bowel sounds. She was diagnosed with acute sigmoid volvulus. Although urgent resective surgery seems to be the appropriate treatment for those who present with acute abdominal pain, intestinal perforation or ischemic necrosis of the intestinal mucosa, the first therapeutic choice for clinically stable patients in good general condition is considered, by many institutions, to be endoscopic decompression. Controversy exists on the decision of the time, the type of definitive treatment, the strategy and the most appropriate surgical technique, especially for teenagers for whom sigmoid resection can be avoided.
\end{abstract}




\section{Introduction}

Acute sigmoid volvulus (SV) is a well-recognized cause of acute large bowel obstruction. Colonic volvulus occurs when the bowel twists on its mesentery, causing obstruction and ischemic changes. The most common location of volvulus is the sigmoid colon $(\sim 60 \%)$, followed by 'cecal' volvulus $(20-40 \%)$ [1]. The differential diagnosis of acute abdominal obstruction includes SV, intussusception, Ogilvie's syndrome, diverticulitis and others $[1,2]$. The most important etiologic factor that has to be considered in adults is that some anatomic lesions are the causative agent $(90 \%)$ and that the majority of these $(65 \%)$ are neoplasms. Other common causes include adenopathy, polyps, diverticulitis and trauma. Many surgeons decide to manage this condition conservatively with colonoscopic decompression alone, usually in elderly people because of co-existing co-morbidities. Nonoperative management of acute SV potentially obviates surgical morbidity in high-risk elderly and frail patients with distended, unprepared bowel. However, since recurrence rates (55-90\%) and mortality rates $(40 \%)$ are high, conservative treatment should be predominantly used in order to move patients from emergency surgery status to semi-elective surgery status $[3,4]$. It is rarely seen in teenagers without any medical history, but when this situation occurs in teenagers, a critical decision remains for the clinical doctor to take: (a) conservative treatment or (b) surgical treatment. We present the case of a teenager with acute SV treated surgically in our department.

\section{Case Report}

A healthy, 18-year-old Caucasian woman presented to the emergency department with a 3-day history of abdominal pain, distention, fecal vomiting, obstipation and constipation. Physical examination revealed decreased bowel sounds, abdominal distention with tympanitic percussion and tenderness. The patient was afebrile and hemodynamically stable. Abdominal X-ray showed a markedly distended sigmoid loop with an inverted U shape, also known as 'coffee bean sign', consistent with SV (fig, 1). Computed tomography (CT) demonstrated a dilated colon with a transitional zone and swirling of the mesentery (fig. 2). A working diagnosis of SV was established. Laboratory data revealed a WBC count of $13,850 / \mathrm{mm}^{3}$ with left shift (86.9\% neutrophils), Ht 37.8\%, LDH $394 \mathrm{U} / \mathrm{l}$, CPK $83 \mathrm{U} / \mathrm{l}, \mathrm{CRP} 7.44 \mathrm{mg} / \mathrm{dl}$ and metabolic acidosis. The patient underwent flexible nasogastric tube insertion, i.v. fluids (lactated Ringer's), i.v. administration of antibiotics (Metronidazole, Amikacin, Cefoxitin), sodium, and food deprivation. Decompression through colonoscopy was not attempted because of the 3 days of pain history. This kind of history is considered to be an absolute contraindication for decompression through colonoscopy because of the risk of existing ischemic colon necrosis, which could lead to bowel perforation during the colonoscopical detorsion attempt. Considering all the facts mentioned above, the patient was taken to the operating room. The findings were a markedly dilated colon (fig. 3 ) (including the right colon), a very redundant mesentery of the left and sigmoid colon, and a counterclockwise SV. The volvulus was located at the rectosigmoid. The sigmoid helices were dilated with $9 \mathrm{~cm}$ diameter. The bowel appeared viable, there was no evidence of ischemia, and the decision was made to perform reduction of the volvulus, sigmoidopexy and, in addition, appendectomy. Biopsies were not taken because of the bad state of the bowel. The operative time was $128 \mathrm{~min}$. Postoperatively, the patient recovered well (fig. 4 ) and regained bowel function on postoperative day 4; the drainage tubes were removed on postoperative day 4 , and she was discharged home on postoperative day 6 .

One year after surgery, the patient presented again to the emergency department with constipation and obstipation. On physical examination the abdomen was distended; abdominal auscultation revealed no bowel sounds. The patient was treated with intravenous fluids and the symptoms subsided within the first day. Afterwards, a diagnostic colonoscopy was performed, in which the patient had not taken any drugs on purpose and did not feel anything during the procedure 
due to the large diameter of the colon. Biopsies were taken from the mucous, but it was not feasible to also take some from the submucosa and the muscularis, in order to obtain samples from the myenteric (Auerbach) and submucosal (Meissner) plexus, which are absent in Hirschsprung disease. Due to this fact we could not specify our clinical judgment as far as Hirsch sprung disease is concerned. The patient was dismissed, given a special diet and is now in an annual follow-up program.

\section{Discussion}

Volvulus of the colon is the leading cause of strangulated large bowel obstruction, with a 33-80\% mortality rate in cases involving intestinal ischemia. Since mortality rates are significantly lower in patients without ischemic change (0-7\%), early diagnosis and treatment are of primary concern.

Unlike many other acute abdomen situations, plain radiographs can often be diagnostic of SV. The key radiologic features of SV are those of a double-loop obstruction, having been reported in $\sim 50 \%$ of patients. The key finding consists of a dilated loop of pelvic colon, associated with features of small bowel obstruction and retention of feces in an undistended proximal colon. The dilated loop usually lies in the right side of the abdomen and the limbs taper inferiorly into the right lower quadrant. Medial deviation of the distal descending colon is a rare but highly specific finding. Single-contrast barium enema examination is usually adequate, if it is required, as in cases when the diagnosis is doubtful. CT scanning is the least invasive imaging technique that allows assessment of mural ischemia. Unlike barium enema examination, CT scanning has a high likelihood of revealing other causes of abdominal pain if the source of the patient's symptoms is not SV. Results of conventional mesenteric angiography with intravenously administered contrast agent or magnetic resonance angiography may be more definitive in the diagnosis of mesenteric ischemia.

The management of SV can be classified into nonsurgical and surgical treatment and includes endoscopic detorsion followed by elective resection, if there is no suspicion of bowel ischemia or perforation [5-10]. Nonoperative techniques, such as decompression through colonoscopy, can convert an emergent procedure in a mentally prepared and medically optimized patient to a semi-elective procedure. Endoscopy provides viability of the bowel, exact location of the damage, mechanical detorsion of the volvulus and decompression of the dilated proximal bowel. Urgent laparotomy is indicated if the suspicion of bowel ischemia is high (e.g. high fever, leukocytosis, peritoneal signs, free abdominal air, acidosis and sepsis). Intraoperative findings and surgeon preference dictate the different surgical alternatives (e.g. colopexy, sigmoid colectomy with primary anastomosis, Hartmann's procedure, Paul-Mikulicz procedure) [3].

The only prospective, randomized study in the literature indicates that sigmoid resection, done either as a Hartmann's procedure when a gangrenous colon is discovered at laparotomy or as a one-stage resection with primary anastomosis in the setting of a viable colon, has the lowest rate of recurrence [10]. Other operative techniques such as sigmoidopexy and, more recently, percutaneous endoscopic colostomy have been used in managing recurrent SV, especially for nongangrenous SV $[7,11]$. There are two objectives in the treatment of SV: the first is to resolve the occlusive phenomena and the second to prevent recurrence of the volvulus [4]. 
The gold standard procedure for a clinically stable patient is decompressive colonoscopy, as long as no signs of peritonitis secondary to ischemia or colon perforation exist, with a rate of effectiveness ranging from 70 to $90 \%$ and a recurrence rate of $18-90 \%$. If the colonoscopy is not effective or if the patient develops signs of peritonitis, urgent surgery is indicated.

Currently, mesosigmoidoplasty is not performed due to the high associated recurrence rates (16-70\%) and mortality (0-11\%). Sigmoidectomy with primary anastomosis is the method of choice for $\mathrm{SV}$, with a mean mortality rate of $8 \%$, a morbidity rate of $13-26 \%$ and a mean recurrence rate of $1.2 \%$. Subtotal colectomy is recommended in order to prevent the recurrence of volvulus in cases of megacolon after the patients have developed recurrence.

The authors believe that healthy teenagers who are diagnosed with SV are a group of patients who would potentially benefit from a minimally and elective surgery approach, as long as the intraoperative findings guarantee that the bowel is viable. It is well known that patients who undergo sigmoidectomy have to get used to a new bowel routine, from severe diarrhea to battle with constipation, weight loss and fecal incontinence. In conclusion, although urgent laparotomy is indicated in acute surgical abdomen, a second thought should be made before performing any kind of colon resection, especially when the patient is young, healthy and mentally stable.

Colonoscopy must be the gold standard procedure for the treatment of acute SV, but when it fails, conservative surgery should be applied. In our case, the patient has not presented SV after 12 months of follow-up and is under observation.

\section{Acknowledgments}

The authors would like to thank their colleagues of the nursing staff of the Surgery Department (NHS), General University Hospital of Alexandroupolis, Greece.

\section{Disclosure Statement}

The authors have no conflicts of interest to declare. 


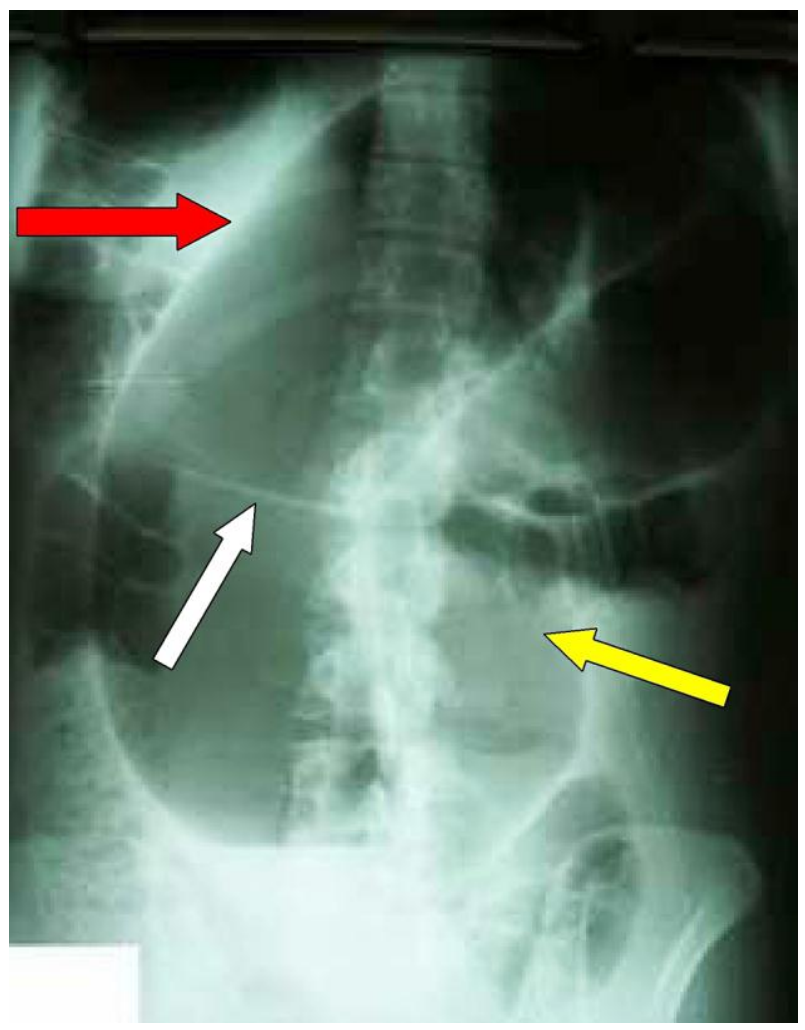

Fig. 1. Abdominal X-ray with a typical 'coffee bean' sign (yellow arrow). The central left of the coffee bean is shown by the white arrow. There is also a liver overlap sign (red arrow) and loss of colonic haustrations. The apex of the sigmoid colon loop is above the level of 10 th thoracic vertebra. The apex of the sigmoid colon loop is under the left dome of the diaphragm.

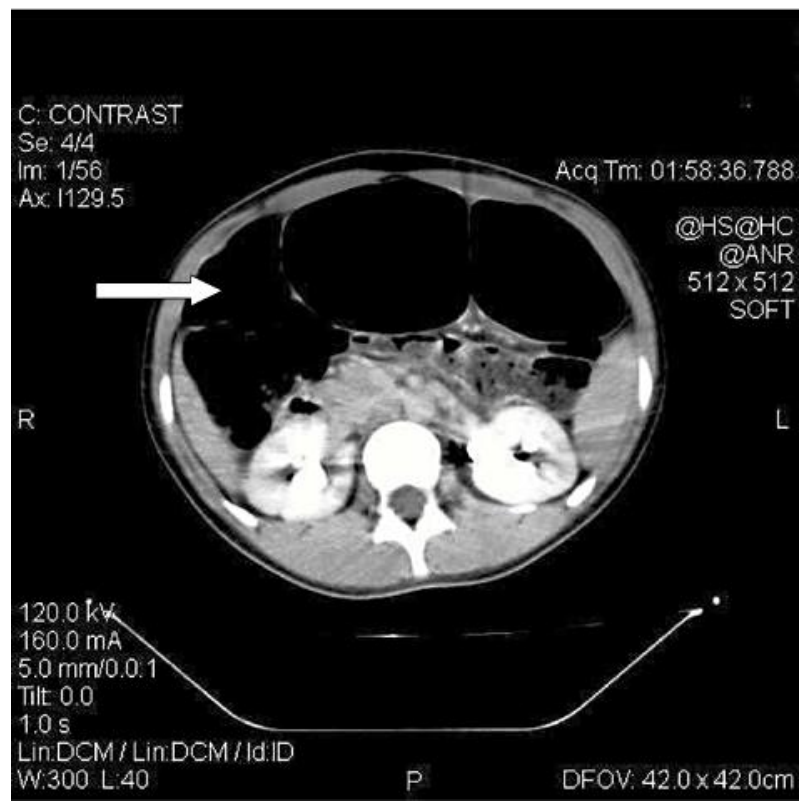

Fig. 2. Abdominal CT image showing sigmoid dilatation, indicating the whirl sign (arrow). 


\begin{tabular}{r|l|l|l}
$\begin{array}{r}\text { Case Reports in } \\
\text { Gastroenterology }\end{array}$ & $\begin{array}{l}\text { Case Rep Gastroenterol 2012;6:293-299 } \\
\text { DOI: 10.1159/000339216 }\end{array}$ & $\begin{array}{l}\text { Published online: } \\
\text { May 23, 2012 }\end{array}$ & $\begin{array}{l}\text { @ 2012 S. Karger AG, Basel } \\
\text { ISSN 1662-0631 } \\
\text { www.karger.com/crg }\end{array}$ \\
\hline
\end{tabular}

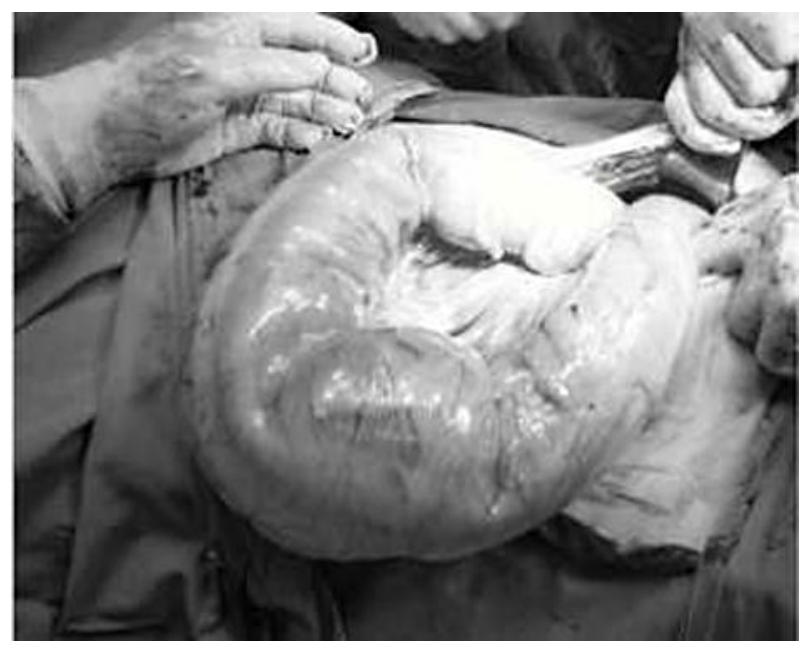

Fig. 3. The patient during surgery.

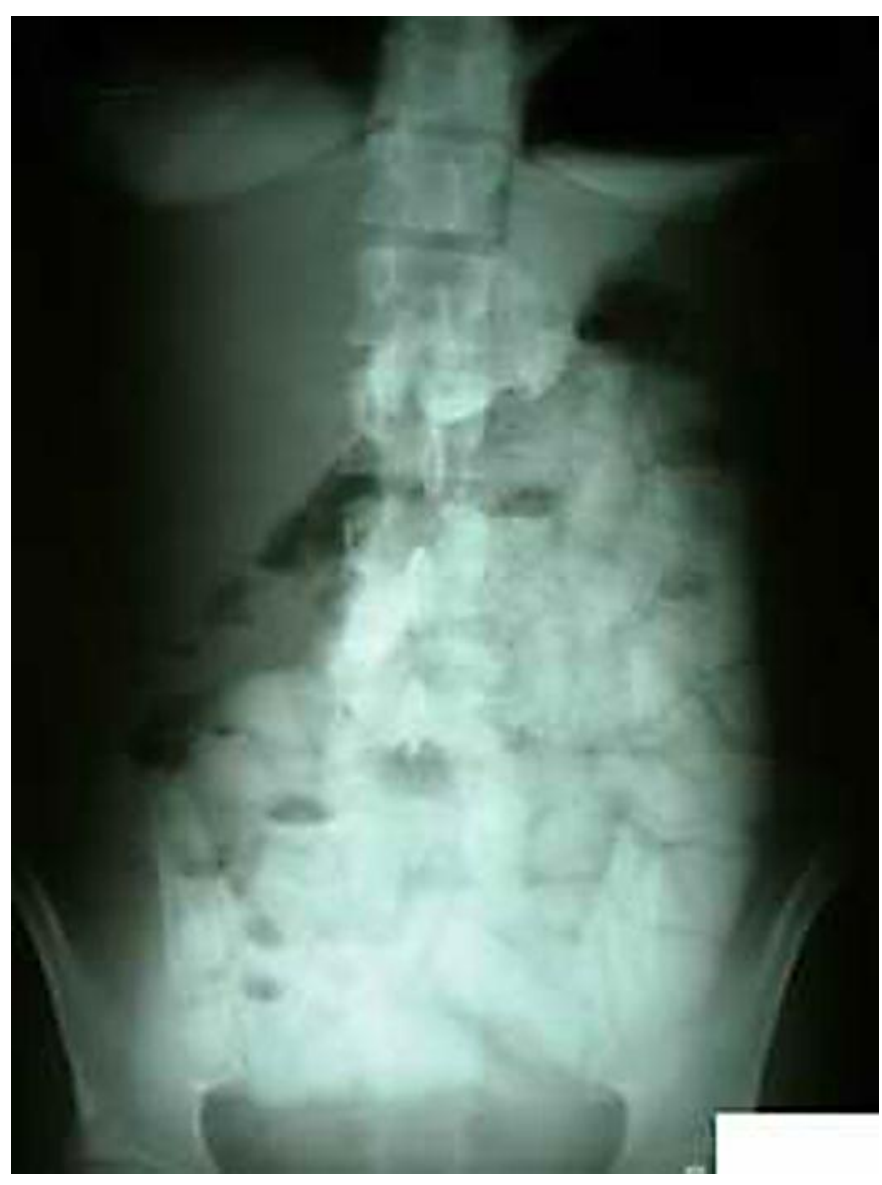

Fig. 4. Abdominal X-ray at follow-up. 


\section{References}

1 Lal SK, Morgenstern R, Vinjirayer EP, Matin A: Sigmoid volvulus an update. Gastrointest Endosc Clin N Am 2006;16:175-187.

-2 Katsikogiannis N, Tsaroucha A, Dimakis K, Sivridis E, Simopoulos C: Rectal endometriosis causing colonic obstruction and concurrent endometriosis of the appendix: a case report. J Med Case Reports 2011;5:320.

3 Kuzu MA, Aslar AK, Soran A, Polat A, Topcu O, Hengirmen S: Emergent resection for acute sigmoid volvulus: results of 106 consecutive cases. Dis Colon Rectum 2002;45:1085-1090.

4 Chung YF, Eu KW, Nyam DC, Leong AF, Ho YH, Seow-Choen F: Minimizing recurrence after sigmoid volvulus. Br J Surg 1999;86:231-233.

5 Oren D, Atamanalp SS, Aydinli B, Yildirgan MI, Basoglu M, Polat KY, Onbas O: An algorithm for the management of sigmoid colon volvulus and the safety of primary resection: experience with 827 cases. Dis Colon Rectum 2007;50:489-497.

-6 Nuhu A, Jah A: Acute sigmoid volvulus in a West African population. Ann Afr Med 2010;9:86-90.

$>7$ Tan KK, Chong CS, Sim R: Management of acute sigmoid volvulus: an institution's experience over 9 years. World J Surg 2010;34:1943-1948.

-8 Jangjoo A, Soltani E, Fazelifar S, Saremi E, Aghaei MA: Proper management of sigmoid colon volvulus: our experience with 75 cases. Int J Colorectal Dis 2010;25:407-409.

-9 Alam MK, Fahim F, Al-Akeely MH, Qazi SA, Al-Dossary NF: Surgical management of colonic volvulus during same hospital admission. Saudi Med J 2008;29:1438-1442.

10 Akcan A, Akyildiz H, Artis T, Yilmaz N, Sozuer E: Feasibility of single-stage resection and primary anastomosis in patients with acute noncomplicated sigmoid volvulus. Am J Surg 2007;193:421-426.

11 Chiang LL, Lai HS, Ni YH, Hsu WM: Management of sigmoid volvulus based on Ladd's procedure: A case report. Pediatr Neonatol 2009;50:129-131. 